\title{
Attributes of Host's Genetic Factors in HIV-1 Pathogenesis
}

\section{Bechan Sharma*}

Professor, Department of Biochemistry, University of Allahabad, Allahabad-211002, UP, India

The human immunodeficiency virus type-1 (HIV-1) is a well known causal organism for AIDS leading to the deaths of millions of people all over the world every year. The anti-HIV-1 regimen available as on today have been designed and developed targeting two key viral enzymes such as (1) HIV-1 reverse transcriptase (HIV-1RT) and (2) protease [1,2]. However, increasing incidences of drugs failures due to emergence of frequent viral mutations as well as toxicity of these compounds in the patients have led to failures of chemotherapy against AIDS [3,4]. Therefore, research into HIV-1 biology is of paramount importance in order to investigate new targets for design and development of novel chemotherapeutics to inhibit not only the wild type virus but also the drug resistant variants [5]. The ongoing research efforts in this direction suggest that HIV-1 exploits a complex net work of many different host cellular factors for the replication of its genome [6,7].

HIV-1 uses host-cellular machinery in order to produce viral genomic material, viral proteins and finally the new mature virions. The hijack and control over host cell processes are mediated by HIV1 proteins through a complex network of molecular events, including virus-host protein-protein interactions (PPIs) [8]. Therefore, by developing our knowledge of the virus-host interaction network, we can improve our current model of HIV-1 infection and host-cell perturbation and use this information to aid development of new antiviral treatments. One example of a successful antiviral treatment that has come from understanding HIV-host cell interaction is the drug maraviroc [9]. Maraviroc is an entry-inhibitor that binds the CCR5 coreceptor, inhibiting gp120:CD4:CCR5 complex formation and, thus, the entry into the host cell $[10,11]$. Targeting a host protein in this way demonstrates that the number of possible HIV-1 therapeutic drug targets is not limited to the small viral proteome and that understanding the virus-host interface can lead to the development of novel-acting therapeutic agents $[12,13]$. The identification of additional cellular cofactors through an independent RNA interference screen has been demonstrated. In fact in last few years, the continued research in this direction has revealed that the cellular factors exhibit dual properties to support as well as to oppose the viral replication; the latter is called as the host restriction factors [14].

After HIV-1 enters the target cell, its cDNA (proviral DNA) integrates into the cellular chromosomes. This process of integration is irreversible in nature and allows HIV-1 to persist in the infected cell in a quiescent or latent stage, which helps the virus to escape from host's immune surveillance as well as antiviral treatment. It is known that both the virus and the host cellular factors are required for HIV-1 replication and expression. Gradually HIV-1 modifies the cellular environment according to its need of efficient replication and production of viral progeny [14]. The viral regulator protein Tat is required for efficient transcription and elongation of viral transcripts. For this purpose, Tat recruits several cellular proteins to make the chromatin structure accessible for the transcription machinery, to acquire the posttranslational modifications essential for its function, and to produce efficient viral replication. In contrast, the host cell also has several restriction factors to encounter viral replication at different steps. The two accessory proteins encoded by HIV-1 namely Vif and
Vpu combat such cellular restrictions and thus significantly contribute in HIV-1 pathogenesis [14].

The different viral proteins which have protein-protein interactions with those of the cellular proteins are as following: The Gag gene of HIV-1 encoded matrix protein (MA) interacts with the cellular Karyopherins, histidyl-tRNA synthetase-like, calmodulin and virion associated nuclear shuttling protein/Nef Associated Factor 1 (VAN/ NAF1); the viral capsid (CA) uses host's Cycophilin A and retroviral restriction factor Tripartite Motif protein (TRIM5a) for uncoating of the virus and its disassembly. This process of uncoating and disassembly of virions also involves another cellular machinery, Arp2/3 complex. The nucleocapsid (NC) protein of HIV-1 interacts with HP68/RNase L inhibitor and actin; the viral p6 protein interacts with many cellular factors such as Tumor susceptibility gene 101 protein (TSG101), ASK1-interacting protein 1 (AIP1), Neuronal precursor cell-expressed developmentally downregulated 4 (Nedd4) and Ubiquitin. Out of the three pol gene encoded viral enzymes (HIV-1RT, protease and integrase), integrase interacts with integrase interactor 1 (INI1/hSNF5), Lens epithelium-derived growth factor/transcription co-activator p75 (LEDGF/p75), ATR, ATM, Karyopherins, BAF and XRCC5 (Ku autoantigen) [15].

The envelope of HIV-1 is comprised of a lipid bilayer derived from the host's cell membrane containing certain proteins which mediate receptor binding and membrane fusion. The glycoproteins present at the viral surface recruit cellular $\mathrm{CD} 4$ receptor which facilitates docking of HIV-1 with CXCR4 or CCR5 co-receptors allowing internalization of the virus. In addition, HIV-1 also may enter into the CD4+ve T-cells via infected dendritic cells (DC) through interaction with DC specific factor, ICAM-3 DC-SIGN. DC-SIGN, a non-integrin, is exclusively expressed by DC, mediates adhesion between DC and resting $\mathrm{T}$ cells via ICAM-3, and is involved in DC-T cell clustering. It is also required for DC-induced proliferation of resting T cells. Upon contact with T-cells, DC helps HIV-1 migrate into T-cells for further infection. Since DC hides HIV-1 as that of macrophages, they evade host's immune surveillance system and cause failures of vaccines [16].

The accessory proteins encoded by HIV-1 such as Nef recruits PACS-1, ASK-1, PAK, P13-Kinase, lck and VAN/NAF1; Rev interacts with Crm 1 and p32; Tat preferably interacts with Cyclin T1; Vpr utilizes karyopherins and Uracil-DNA glycosylase; and the viral Vif interacts

*Corresponding author: Bechan Sharma, Professor, Department of Biochemistry, University of Allahabad, Allahabad-211002, UP, India, Tel: +91-9415715639; E-mail: sharmabi@yahoo.com

Received June 02, 2012; Accepted June 05, 2012; Published June 08, 2012

Citation: Sharma B (2012) Attributes of Host's Genetic Factors in HIV-1 Pathogenesis. Biochem Anal Biochem 1:e109. doi:10.4172/2161-1009.1000e109

Copyright: (C) 2012 Sharma B. This is an open-access article distributed under the terms of the Creative Commons Attribution License, which permits unrestricted use, distribution, and reproduction in any medium, provided the original author and source are credited. 
with host's APOBEC3G (A3G) and helps in its disintegration in order to avoid $\mathrm{A} 3 \mathrm{G}$ mediated hypermutation into proviral DNA. The $\mathrm{A} 3 \mathrm{G}$ is also involved in uncoating and the disassembly of the virus [17].

The reverse transcription process of HIV-1 genome is essential to synthesize proviral cDNA. The heterodimeric (p66/p51) reverse transcriptase (RT) utilizes cellular tRNALys3 as a primer containing about 18 nucleotides from its 3 '-end which are complementary to the viral genomic RNA. It anneals with the primer binding site located at the 5 ' terminus of viral genomic LTR region and provides 3 '- $\mathrm{OH}$ group for synthesis of first (-) single stranded DNA (ssDNA). The other cellular factors involved at different stages of reverse transcription in HIV-1 are actin, tubulin and karyopherin $[17,18]$.

The integrase interactor 1INI1/hSNF5, a component of SNF-SWI Complex, facilitates nuclear import of the pre-integration complex (PIC) as well as the integration reaction. INI1 is also needed at the late events of viral life cycle. The binding of human lens epitheliumderived growth factor / transcription co-activator p75 (LEDGF/p75) with integrase is crucial in nuclear import of PIC. HMGal and BAF have also been shown to play key role in this process $[17,19,20]$. A detailed account of host cell factors that inhibit or assist viral DNA integration processes is reviewed by Sloan and Wainberg [21]. Many of those factors involved in cellular DNA repair processes such as XPB and XPD which are DNA helicases and part of TFII B transcription complex play role in DNA nucleotide excision repair [22]. AtaxiaTelangiectasia Mutated (ATM) is especially critical for repair of DSBs, whereas ATM- and Rad3-related (ATR) kinase appears to be specific for lesions that contain single strands coated with replication protein A (RPA) $[19,23]$.

The P6 domain within HIV-1 gag is involved in budding in association with the host cellular protein, TSG101. HIV-1 release requires TSG101 that sorts proteins into vesicles that bud into multivesicular bodies (MVB) [17,24]. TSG101+PTAP motif of p6 complex recruits ESCRT III (endosome associated complex required for transport) to the budding site. Similar to TSG101, the multifunctional proteins AIP1/ALIX also bind to the $\mathrm{p} 6$ and plays role in pinch-off process. Lysosomal degradation of ubiquitinated receptors is directed by the sequential action of the ubiquitin-binding protein complex composed of Hrs, TSG101 and ALIX with associated proteins. The endocytic sorting machinery is recruited during the budding of RNA viruses, whose Gag proteins are ubiquitinated and have motifs that bind TSG101 and ALIX [25].

Apolipoprotein B m-RNA-editing enzyme-catalytic polypeptidelike $3 \mathrm{G}$ (APOBEC3G) can deaminate $\mathrm{C}$ to yield $\mathrm{U}$ on the minus-strand of viral genome, which causes G-to-A conversion on the complimentary plus strand. The G-to-A hypermutation alters the nucleotide sequences of the viral genome leading to inability in HIV-1 replication. The viral accessory protein, Vif, binds to APOBEC3G to induce proteasomedependent degradation; thereby it is not incorporated into HIV-1 particles. Thus Vif protects the viral genome by this mechanism. The Vif-mediated ubiquitination / degradation of APOBEC3G involve four critical lysine residues in its C-terminal domain [17,26,27]. However, cellular Cycophilin A (CypA) protects HIV-1 from an unknown antiviral activity in human cells. It regulates the processes of internalization as well as assembly of HIV-1[28].

The viral accessory protein Nef plays an important role in HIV-1 pathogenesis by interacting with various cellular proteins; one of them being Cyclin $\mathrm{K}(\mathrm{CycK})$. CycK acts as a cellular restriction factor and inhibits HIV-1 gene expression and replication in a Nef-dependent manner [29]. Further, the role of cellular protein kinase C (PKC) theta $(\theta)$ as another host's restriction factor opposing the viral replication has been indicated [30]. Unlike the roles of CycK and PKC $\theta$, one of the cellular soluble factor, tumor necrosis factor-alpha (TNF- $\alpha$ ) enhances

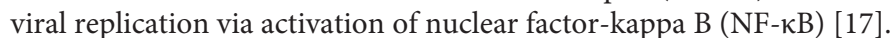
The increasing knowledge of viral protein interactions with host cell factors would be essential for the discovery of new targets that could be used to design new therapeutic strategies [14].

\section{References}

1. Ferrantelli F, Cafaro A, Ensoli B (2004) Nonstructural HIV proteins as targets for prophylactic or therapeutic vaccines. Curr Opin Biotechnol 15: 543-556.

2. Ryser HJ, Flückiger R (2005) Progress in targeting HIV-1 entry. Drug Discov Today 10: 1085-1094.

3. Carr A (2003) Toxicity of antiretroviral therapy and implications for drug development. Nat Rev Drug Discov 2: 624-634.

4. Sharma B (2011) Anti-HIV-1 drugs toxicity and management strategies Neurobehavioural HIV Medicine 3: 27-40.

5. Reeves JD, Piefer AJ (2005) Emerging drug targets for antiretroviral therapy. Drugs 65: 1747-1766.

6. Bean P (2005) New drug targets for HIV. Clin Infect Dis 41: 96-100.

7. Flexner C (2007) HIV drug development: the next 25 years. Nat Rev Drug Discov 6: 959-966.

8. Pinney JW, Dickerson JE, Fu W, Sanders-Beer BE, Ptak RG, et al. (2009) HIV-host interactions: a map of viral perturbation of the host system. AIDS 23 549-554.

9. Dorr P, Westby M, Dobbs S, Griffin P, Irvine B, et al. (2005) Maraviroc (UK$427,857)$, a potent, orally bioavailable, and selective small-molecule inhibitor of chemokine receptor CCR5 with broad-spectrum anti-human immunodeficiency virus type 1 activity. Antimicrob Agents Chemother 49: 4721-4732.

10. Biswas P, Tambussi G, Lazzarin A (2007) Access denied? The status of coreceptor inhibition to counter HIV entry. Expert Opin Pharmacother 8: 923-933.

11. Levy JA (2009) HIV pathogenesis: 25 years of progress and persistent challenges. AIDS 23: 147-160.

12. Zhu Y, Chen G, Lv F, Wang X, Ji X, et al. (2011) Zinc-finger antiviral protein inhibits HIV-1 infection by selectively targeting multiply spliced viral mRNAs for degradation. Proc Natl Acad Sci USA 108:15834-15839.

13. Cherrier T, Elias M, Jeudy A, Gotthard G, Le Douce V, et al. (2011) HumanPhosphate-Binding-Protein inhibits HIV-1 gene transcription and replication. Virol J 8: 352.

14. Coiras M, López-Huertas MR, Sánchez del Cojo M, Mateos E, Alcamí J (2010) Dual Role of Host Cell Factors in HIV-1 Replication: Restriction and Enhancement of the Viral Cycle. AIDS Rev 12: 103-112.

15. Tastan O, Qi Y, Carbonell JG, Kleinseetharaman J (2009) Prediction of interactions between HIV-1 and human proteins by information integration. Pac Symp Biocomput 516-527.

16. Evans P, Dampier W, Ungar L, Tozeren A (2009) Prediction of HIV-1 virus-host protein interactions using virus and host sequence motifs. BMC Med Genomics 2: 27 .

17. Komano J, Futahashi Y, Urano E, Miyauchi K, Murakami T, et al. (2005) The interaction of HIV-1 with host factors. Jpn J Infect Dis 58: 125-130.

18. Kvaratskhelia M, Miller JT, Budihas SR, Pannell LK, Le Grice SF (2002) Identification of specific HIV-1 reverse transcriptase contacts to the viral RNA:tRNA complex by mass spectrometry and a primary amine selective reagent. Proc Natl Acad Sci USA 99: 15988-15993.

19. Engelman A, Cherepanov $P(2008)$ The lentiviral integrase binding protein LEDGF/p75 and HIV-1 replication. PLoS Pathog 4: 1000046.

20. Pluta K, Kacprzak MM (2009) Use of HIV as a gene transfer vector. Acta Biochim Pol 56: 531-595.

21. Sloan RD, Wainberg MA (2011) The role of unintegrated DNA in HIV infection Retrovirology 8: 52. 
Citation: Sharma B (2012) Attributes of Host's Genetic Factors in HIV-1 Pathogenesis. Biochem Anal Biochem 1:e109. doi:10.4172/21611009.1000e109

Page 3 of 3

22. Deng W, Roberts SG (2007) TFIIB and the regulation of transcription by RNA polymerase II. Chromosoma 116: 417-429.

23. Skalka AM, Katz RA (2005) Retroviral DNA integration and the DNA damage response. Cell Death Differ 12: 971-978.

24. von Schwedler UK, Stuchell M, Müller B, Ward DM, Chung HY, et al. (2003) The protein network of HIV budding. Cell 114: 701-713.

25. Szymkiewicz I, Shupliakov O, Dikic I (2004) Cargo- and compartment-selective endocytic scaffold proteins. Biochem J 383: 1-11.

26. Goncalves J, Santa-Marta M (2004) HIV-1 Vif and APOBEC3G: Multiple roads to one goal. Retrovirology 1: 28.

27. Yu X, Yu Y, Liu B, Luo K, Kong W, et al. (2003) Induction of APOBEC3G
Ubiquitination and Degradation by an HIV-1 Vif-Cul5-SCF Complex. Science 302: $1056-1060$

28. Braaten D, Luban J (2001) Cyclophilin A regulates HIV-1 infectivity, as demonstrated by gene targeting in human T cells. EMBO J 20: 1300-1309.

29. Khan SZ, Mitra D (2011) Cyclin K inhibits HIV-1 gene expression and replication by interfering with cyclin-dependent kinase 9 (CDK9)-cyclin T1 interaction in Nef-dependent manner. J Biol Chem 286: 22943-22954.

30. López-Huertas MR, Mateos E, Díaz-Gil G, Gómez-Esquer F, del Cojo MS, et al. (2011) Protein Kinase $C$ (PKC) theta $(\theta)$ is a specific target for the inhibition of the human immunodeficiency virus type 1 (HIV-1) replication in CD 4+ lymphocytes. Journal of biological chemistry M110.210443. 